\title{
Potential of S-Allyl Cysteine, a Major Bioactive Component of Garlic, As Hypoglycemic and Hypolipidemic Agent
}

\author{
Nadeem Rais ${ }^{1 *}$, Akash Ved ${ }^{2}$, Rizwan Ahmad ${ }^{3}$ and Kehkashan Parveen ${ }^{4}$ \\ ${ }^{1}$ Department of Pharmacy, Bhagwant University, India \\ ${ }^{2}$ Goel Institute of Pharmaceutical Sciences, India \\ ${ }^{3}$ Department of Pharmacy, Vivek College of Technical Education, India \\ ${ }^{4}$ Interdisciplinary Biotechnology Unit, Aligarh Muslim University, India \\ Submission: April 20, 2021; Published: May 18, 2021 \\ *Corresponding author: Nadeem Rais, Department of Pharmacy, Bhagwant University, Ajmer, Rajasthan, 305004, India
}

Abstract

Diabetes Mellitus (DM) is a global health issue characterized by hyperglycemia, insulin resistance, absolute or relative impairment in insulin secretion, action or both. Diabetes and insulin resistance have a close association with body mass index, and the increasing occurrence of DM is linked to weight gain and body mass. Treatment options are very unsatisfactory having multiple adverse effects. S-Allyl cysteine (SAC) is an organic compound and a natural constituent of Allium sativum, commonly known as garlic, and is widely acknowledged to have a variety of biological activities with minimal side effects. The aim of this study was to review the hypoglycemic and hypolipidemic effects of SAC, a sulfurcontaining amino acid.

Keywords: Diabetes; Obesity; S-allyl cysteine; Garlic; Adipose tissue; Antioxidants

Abbreviations: DM: Diabetes Mellitus; BMI: Body Mass Index; SAC: S-Allyl Cysteine; T2DM: Type 2 Diabetes Mellitus; AGE: Aged Garlic Extract

\section{Introduction}

DM is a category of diseases characterized by elevated blood glucose levels due to insulin secretion, insulin action, or both. There are also abnormalities in carbohydrate, protein, and fat metabolism [1]. Obesity has played a significant role in the diabetes crisis, with almost $80 \%$ of people with T2DM being overweight or obese [2]. The adoption and maintenance of healthy lifestyle habits, such as those outlined in the National Diabetes Prevention Program, will undoubtedly help to avoid or delay T2DM [3]. Obesity and T2DM have become more common around the world in the last century, not just in developed countries but also in developing countries, often coexisting with malnutrition. Moreover, the global prevalence of DM was 280 million people in 2010 (around 6.2 percent of the global population), and it is estimated that by 2030, the prevalence will have risen to more than 7.5 percent of the global population, paralleling population ageing and BMI, reinforcing the connection between obesity and diabetes [4].
Obesity and diabetes are conditions that can shorten life expectancy, reduce quality of life, and drive up healthcare costs. Obesity and diabetes rates are continuing to increase at epidemic levels. The new word "diabesity" was originally used to describe diabetes caused by obesity. Obese people are more likely to develop T2DM, also known as insulin-resistant diabetes or adultonset diabetes. This is a disorder in which the blood glucose level remains high for an extended period of time. Obesity is believed to cause alterations in the body's metabolism. Fat tissue (adipose tissue) releases fat molecules into the bloodstream as a result of these modifications, which can impact insulin-responsive cells and reduce insulin sensitivity [5]. Around 25\% to 30\% of type 2 diabetic patients do not react to oral agents (primary failures), whereas $5 \%$ of those who did respond initially become nonresponsive to these agents (Secondary failures). Aside from that, the available medications have a slew of negative side effects on the human body. Scientists are working constantly to find new 


\section{Current Research in Diabetes \& Obesity Journal}

agents to help solve this global problem. In prehistoric times, plants were used as medicine and as a source of medicine [6].

Garlic (Allium sativum, Liliaceae) has long been used as a complementary therapy for a variety of diseases, including diabetes, cancer, and neurodegenerative disorders including ischemic stroke. Garlic has also been shown to have a number of cardiovascular benefits, including reducing plasma cholesterol, inhibiting platelet aggregation, and dropping arterial blood pressure.

Garlic contains hundreds of phytochemicals, including sulfurcontaining substances like ajoenes (E \& Z), allicin, sulphides (diallyl disulfide - DADS, diallyl trisulfide - DATS), and others, which account for 82 percent of the total sulfur content (Table 1). After chopping off the garlic and breaking down the parenchyma, alliin, the dominant cysteine sulfoxide, is converted to allicin by the allinase enzyme. Garlic formulations include organosulfur compounds derived from alliin, such as $\mathrm{N}$-acetylcysteine (NAC), S-allyl-cysteine (SAC), and S-allyl-mercapto cysteine (SAMC). SAC is an organosulfur-containing amino acid that is a major bioactive component of garlic. SAC, like garlic extract, is said to have antioxidant, anti-diabetic, anti-obesity, anti-cancer, antihepatotoxic, and anti-stroke properties [7].

Table 1: List of some sulfur-containing compounds of garlic (Allium sativum) with their molecular formula.

\begin{tabular}{|c|c|}
\hline Garlic Compounds & Molecular Formula \\
\hline Alliin (S-allyl-L-cysteine sulfoxide) & $\mathrm{C}_{6} \mathrm{H}_{11} \mathrm{NO}_{3} \mathrm{~S}$ \\
\hline Allicin [S-(2-propenyl)-2-propene-1-sulfinothioate] & $\mathrm{C}_{6} \mathrm{H}_{10} \mathrm{OS}_{2}$ \\
\hline Z-Ajoene & $\mathrm{C}_{9} \mathrm{H}_{14} \mathrm{OS}_{3}$ \\
\hline E-Ajoene & $\mathrm{C}_{9} \mathrm{H}_{14} \mathrm{OS}_{3}$ \\
\hline Diallyl sulfide (DAS) & $\mathrm{C}_{6} \mathrm{H}_{10} \mathrm{~S}$ \\
\hline Diallyl disulfide (DADS) & $\mathrm{C}_{6} \mathrm{H}_{10} \mathrm{~S}_{2}$ \\
\hline Diallyl trisulfide (DATS) & $\mathrm{C}_{6} \mathrm{H}_{10} \mathrm{~S}_{3}$ \\
\hline S-allyl cysteine (SAC) & $\mathrm{C}_{6} \mathrm{H}_{11} \mathrm{NO}_{2} \mathrm{~S}$ \\
\hline
\end{tabular}

\section{Garlic Preparations}

Essential oil, dehydrated powder, oil macerate, and extract are some of the garlic preparations. Garlic powders (tablets), garlic oils (capsules), and aged garlic extracts (tablets, capsules, and liquid) are among the commercially available garlic supplements; each one of these sources offers a different profile of organosulfur compounds. Aged Garlic Extract (AGE) is a wellstudied garlic preparation [8]. In stainless steel tanks, raw garlic slices are aged for up to 20 months (in 15-20 percent ethanol). After that, the extract is filtered before being concentrated at a low temperature. AGE is stated to possess numerous biological properties which include antioxidant, anti-inflammatory, neuroprotective, anti-neoplasic, anti-obesity, anti-diabetic, antiallergic, cardioprotective, and hepatoprotective effects. AGE is available in two forms: dry powder and a liquid of $10 \%$ ethanol. This whole process is expected to result in a significant loss of allicin and an increase in the activity of newer compounds such as SAC (Figure 1), which is stable, highly bioavailable, least toxic than other garlic compounds, and a largely abundant organosulfur compound extracted from garlic [8,9]. Garlic contains a variety of similar compounds, including the disulfide SAMC and $\gamma$-GlutamylS-allyl cysteine (GSAC). SAC content in integral garlic is low, but this component is produced in a dripping by hydrolysis of GSAC (via $\gamma$-Glutamyltransferase), a SAC originator found in unrefined garlic (Figure 2) [9].<smiles>C=CCSCC(N)C(=O)O</smiles>

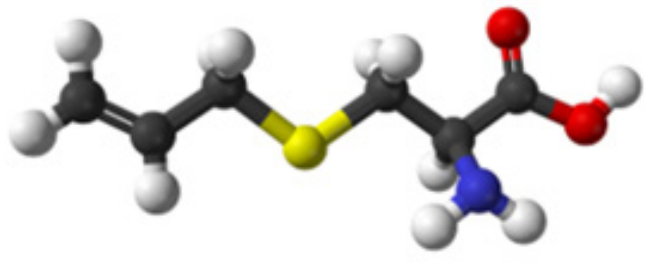

Figure 1: Structure of S-allyl cysteine $\left(\mathrm{C}_{6} \mathrm{H}_{11} \mathrm{NO}_{2} \mathrm{~S}\right)$ 


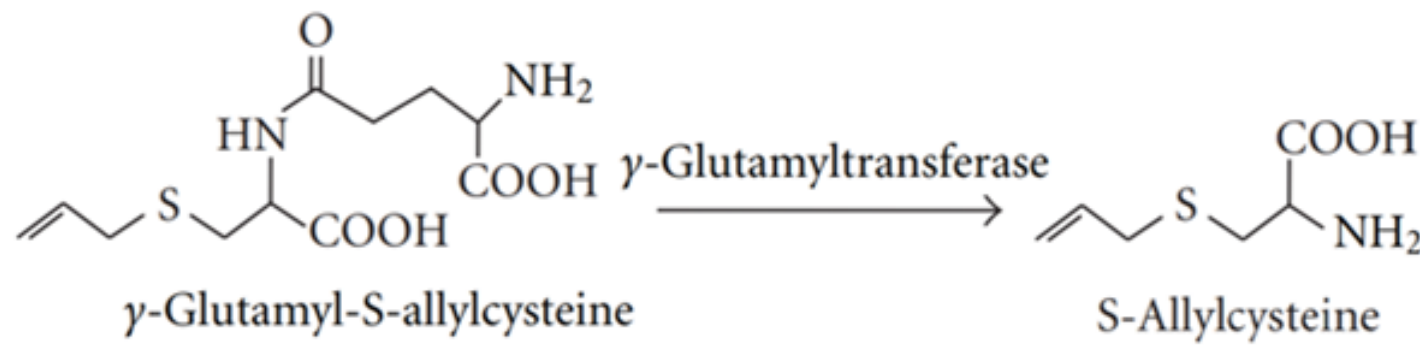

Figure 2: $\mathrm{Y}$-Glutamyltransferase converts $\mathrm{\gamma}$-glutamyl-S-allyl cysteine to S-allyl cysteine (SAC) during the ageing process (figure adapted from Colín-González et al. [9]).

\section{Physicochemical properties of SAC}

SAC (synonym: S-allyl-laevo-cysteine) is a water-soluble white crystalline powder with a distinct odour. Its melting point is 219 to $220^{\circ} \mathrm{C}$ ( 426 to $428{ }^{\circ} \mathrm{F}$ ), molecular weight is $161.22 \mathrm{~g} / \mathrm{mol}$, and its molecular formula is C6H11NO2S. SAC is a stable compound since it can last up to two years in AGE without changing. The colour of stored crystal samples change slightly to a yellowish shade, but no transformation or decomposition is occurred. These findings suggest that after oral administration, SAC can be rapidly absorbed in the gastrointestinal tract without causing any changes. SAC is 30 times less toxic (LD50 value $>20 \mathrm{mM} / \mathrm{kg}$ i.p.; $>54.7 \mathrm{mM} / \mathrm{kg}$ p.o.) than other common garlic compounds like allicin and diallyldisulfide [7-10].

\section{Scavenger characteristics of SAC}

SAC is by far the most abundant component in aged garlic extract, and it has potent antioxidant properties. SAC treatment can reduce reactive oxygen species (ROS), such as superoxide radical and hydrogen peroxide (H2O2), at the cellular level. SAC can scavenge superoxide radicals and reduce the development of hydrogen peroxide $[7,11]$. SAC has been shown in many studies to be a stable, water-soluble compound that lowers cholesterol, acts as an antioxidant, prevents cancer, and protects the liver from toxins. Since it is measurable and rises quantitatively in the blood after oral ingestion of garlic capsules, SAC is currently the only accurate human compliance marker used in experiments concerning garlic consumption [12]. The potentiality of sulfur containing amino acid (SAC) derived from garlic on DM and other disorder was investigated in a report. SAC improve glucose and lipid metabolism, reform hyperglycemia, insulin resistance, hyperlipidemia, and convalesce adipose tissue metabolism, as well as oxidative stress production and processes of stress-sensitive signalling and inflammatory related issues, as shown by a variety of in vitro, animal studies, and some human clinical trials [13].

\section{SAC as hypoglycemic agent}

Normal and STZ-diabetic rats were given the SAC orally (150 $\mathrm{mg} / \mathrm{kg} \mathrm{bw}$ ) for 45 days. The blood glucose levels of the diabetic rats increased. Furthermore, diabetic rats had lower activity of hexokinase, glycogen synthase, and liver glycogen, while having higher activity of enzymes including glucose-6-phosphatase, fructose-1,6-bisphosphatase, and glycogen phosphorylase. SAC treatment resulted in a substantial reduction in blood glucose and significantly reduced these enzyme behaviors. As a result, the findings indicate that SAC has antihyperglycemic properties and support its conventional use in diabetes management [10].

For 45 days, STZ-induced diabetic rats were given SAC at two separate doses (100 mg/kg b.w. and $150 \mathrm{mg} / \mathrm{kg}$ b.w.). SAC treatment reduced blood glucose, glycosylated haemoglobin, blood urea, serum uric acid, and serum creatinine levels significantly, and pathophysiological enzymes such as aspartate transaminase (AST), alanine transaminase (ALT), and alkaline phosphatase (ALP) were also diminished. SAC's antihyperglycaemic properties are also demonstrated by improvements in plasma insulin and haemoglobin levels. Furthermore, the findings are similar to those obtained with glyclazide, an oral standard medication. A dose of $150 \mathrm{mg} / \mathrm{kg}$ b.w. had a greater effect than a dose of $100 \mathrm{mg} / \mathrm{kg}$ b.w. As a result of the current findings, SAC could be considered a reliable therapeutic option for the management of diabetes [14].

The oral administration of SAC (150 mg/kg b.w.) to diabetes rats reduced the levels of glycoprotein components in the liver. The potentiation of insulin discharge from $\beta$-cells of the Langerhan's islets, which can grow due to glucose dissemination, may be one mechanism by which SAC achieves glycoprotein compound standardization. Reduced glycoprotein levels in the liver, kidney, and plasma may be responsible for the reduced hyperglycemic condition in SAC-treated diabetic rats. It has been reported that when SAC is administered, it reverses the negative effects of DM, thereby intruding into the pathogenesis of diabetic complications, and could be used to benefit in therapeutical purposes [15].

\section{SAC as hypolipidemic agent}

Hyperlipidemia is a complication of diabetes. The link between diabetes and lipid parameters changes is a major risk factor for cardiovascular complications in diabetics. Adipose tissue depots 


\section{Current Research in Diabetes \& Obesity Journal}

undergo significant hypertrophy in obesity, as well as the secretion of adipokines and proinflammatory cytokines by both adipocytes and infiltrating immune cells, resulting in low-grade inflammation and the long-term production of insulin resistance [16].

Several therapies for the treatment of obesity and its comorbidities have been released in recent years. However, because of their negative impact, the majority of them have been taken off the market [17]. As a result, several pharmaceutical firms are focused on developing a new medication with no side effects. Among natural phytochemical compounds, aged black garlic (ABG) has been shown to prevent some of the cardiometabolic changes associated with metabolic syndrome in both humans and laboratory animals in several studies [18].

Hyperlipidemia and normal coronary function have an opposite relationship. As a result, the primary treatment for alleviating coronary heart disease is lowering elevated serum cholesterol levels. Hyperlipidemia was induced in the current study using a high fat diet that included dalda (30\%), a saturated fat, and pure cholesterol (2\%) in a regular diet. The animals were fed this diet for two weeks before receiving the planned medications, AGE and SAC. There was an increase in TG (triglyceride), LDL (lowdensity lipoproteins) cholesterol, and TC (total cholesterol) levels without a change in HDL (high-density lipoproteins) cholesterol levels. Treatment with AGE and SAC reduced serum TG levels. This tendency may be defined as a significant release of endotheliumbound lipoprotein lipase, which hydrolyses TG into fatty acids, mediated by AGE and SAC [19].

Treatment with SAC normalized elevated levels of LDL, TG, VLDL (very low density lipoproteins) cholesterol, serum TC, and decreased HDL cholesterol. This hypolipidemic activity may be accomplished by an increase in insulin secretion, which leads to a decrease in fatty acid and cholesterol synthesis. Diabetic rats given SAC (150 mg/kg b.w.) showed a decrease in liver triglycerides, phospholipids, cholesterol, and free fatty acids. This may be due to improved consent and decreased fabrication of the main transporters that manufacture triglycerides and cholesterol endogenously. When SAC was given to diabetic rats, the concentration of these lipids was found to be lower in diabetes treated rats when compared to diabetes control rats [20].

In another analysis, both AGE and SAC reduced TC and LDL while significantly increased HDL, demonstrating its superiority to other hypolipidemics. The hypolipidemic potential of AGE and SAC is attributed to a $41 \%$ deactivation of 3-hydroxy-3methylglutaryl-CoA [21].

\section{Conclusion}

A number of studies have found a clear link between obesity and the development of diabetes. According to reports, people who are overweight are more insulin resistant and have a harder time controlling their diabetes. This review concludes that S-allyl cysteine, a major bioactive component of garlic, has the potential to act as hypoglycemic and hypolipidemic agent with minimal adverse effects. SAC administration improves the metabolic and vascular alterations. Further, the exact mechanism of SAC should be discovered in future studies.

\section{Acknowledgement}

The authors are thankful to Prof. (Dr.) Saurabh Sharma, Principal, Department of Pharmacy, Vivek College of Technical Education, Bijnor, Uttar Pradesh, India for his sustained encouragement, meticulous supervision, and valuable suggestions at all stages of completion of this manuscript.

\section{References}

1. American Diabetes Association (2021) Obesity Management for the Treatment of Type 2 Diabetes: Standards of Medical Care in Diabetes-2021. Diabetes Care 44(Suppl 1): S100-S110.

2. Wander PL, Boyko EJ, Leonetti DL, McNeely MJ, Kahn SE, et al. (2013) Change in visceral adiposity independently predicts a greater risk of developing type 2 diabetes over 10 years in Japanese Americans. Diabetes Care 36(2): 289-293.

3. Ali MK, Echouffo-Tcheugui J, Williamson DF (2012) How effective were lifestyle interventions in real-world settings that were modeled on the Diabetes Prevention Program? Health Aff (Millwood) 31(1): 67-75.

4. Yang W, Lu J, Weng J, Jia W, Ji L, et al. (2010) China National Diabetes and Metabolic Disorders Study Group. Prevalence of diabetes among men and women in China. N Engl J Med 362(12): 1090-1101.

5. Al-Goblan AS, Al-Alfi MA, Khan MZ (2014) Mechanism linking diabetes mellitus and obesity. Diabetes Metab Syndr Obes 7: 587-591.

6. Ansari MA, Phull QZ, Arain AA, Memon AR, Kazi S (2017) Comparison between S-Allyl Cysteine and Gliclazide in Lowering the Blood Glucose Levels in Diabetic Rats. J Liaquat Uni Med Health Sci 16(02): 99-102.

7. Numagami Y, Ohnishi ST (2001) S-allylcysteine inhibits free radical production, lipid peroxidation and neuronal damage in rat brain ischemia. J Nutr 131(3s): 1100S-1105S.

8. Banerjee SK, Maulik SK (2002) Effect of garlic on cardiovascular disorders: a review. Nutr J 1: 4.

9. Colín-González AL, Santana RA, Silva-Islas CA, Chánez-Cárdenas ME Santamaría A, et al. (2012) The antioxidant mechanisms underlying the aged garlic extract- and S-allylcysteine-induced protection. Oxid Med Cell Longev 2012: 907162.

10. Saravanan G, Ponmurugan P, Senthilkumar GP, Rajarajan T (2009) Modulatory effect of S-allylcysteine on glucose metabolism in streptozotocin induced diabetic rats. J Funct Foods 1(4): 336-340.

11. Kim JS, Park SK (2017) Supplementation of S-allyl cysteine improves health span in Caenorhabditis elegans. Biosci J 33(2): 411-421.

12. Amagase H, Petesch BL, Matsuura H, Kasuga S, Itakura Y (2001) Intake of garlic and its bioactive components. J Nutr 131(3s): 955S-962S.

13. Sathibabu-Uddandrao VV, Brahmanaidu P, Saravanan G (2017) Therapeutical Perspectives of S-Allylcysteine: Effect on diabetes and other disorders in Animal Models. Cardiovasc Hematol Agents Med Chem 15(2): 71.

14. Saravanan G, Ponmurugan P, Senthil kumar GP, Rajarajan T (2009) Antidiabetic properties of S-allylcysteine, a garlic component on streptozotocin-induced diabetes in rats. J Appl Biomed 7: 151-159. 


\section{Current Research in Diabetes \& Obesity Journal}

15. Saravanan G, Ponmurugan P, Senthil Kumar GP, Rajarajan T (2010) Antidiabetic effect of S-allylcysteine: Effect on plasma and tissue glycoproteins in experimental diabetes. Phytomedicine 17(14): 10861089.

16. Pirola L, Ferraz JC (2017) Role of pro- and anti- inflammatory phenomena in the physiopathology of type 2 diabetes and obesity. World J Biol Chem 8(2): 120-128.

17. Kang JG, Park CY (2012) Anti-Obesity Drugs: A Review about Their Effects and Safety. Diabetes Metab J 36(1): 13-25.

18. Ryu JH, Kang D (2017) Physicochemical Properties, Biological Activity, Health Benefits, and General Limitations of Aged Black Garlic: A Review. Molecules 22(6): 919.
19. Devi R, Sharma DK (2004) Hypolipidemic effect of different extracts of Clerodendron colebrookianum Walp in normal and high-fat diet fed rats. J Ethnopharmacol 90(1): 63-68.

20. Saravanan G, Ponmurugan P (2012) Ameliorative potential of S-allylcysteine: effect on lipid profile and changes in tissue fatty acid composition in experimental diabetes. Exp Toxicol Pathol 64(6): 639644.

21. Asdaq SM (2015) Antioxidant and hypolipidemic potential of aged garlic extract and its constituent, s-allyl cysteine, in rats. Evid Based Complement Alternat Med 2015: 328545.

Your next submission with Juniper Publishers
will reach you the below assets
- Quality Editorial service
- Swift Peer Review
- Reprints availability
- E-prints Service
- Manuscript Podcast for convenient understanding
- Global attainment for your research
- Manuscript accessibility in different formats
( Pdf, E-pub, Full Text, Audio)
- Unceasing customer service
Track the below URL for one-step submission
https://juniperpublishers.com/online-submission.php

